\title{
Projecting the Long-Term Economic Impact of Once-Monthly Paliperidone Palmitate Versus Oral Atypical Antipsychotics in Medicaid Patients with Schizophrenia
}

\author{
Antoine C. El Khoury, PhD; Dominic Pilon, MA; Laura Morrison, MSc; Nina Shak, BA; \\ Amanda Llaneza, BA; Edward Kim, MD, MBA; and Patrick Lefebvre, MA
}

\begin{abstract}
BACKGROUND: Recent evidence has demonstrated that, over 12 months, pharmacy costs associated with switching nonadherent recently relapsed patients from oral atypical antipsychotics (OAAs) to once-monthly paliperidone palmitate (PP1M) were offset by reduced relapse rates and schizophrenia-related health care costs. In addition, earlier use of PP1M may generate greater cost savings.
\end{abstract}

OBJECTIVE: To project the long-term economic impact when a proportion of nonadherent patients with a recent relapse switch from OAAs to PP1M.

METHODS: A 36-month decision-tree model with twelve 3-month cycles was developed from a Medicaid payer's perspective. The target population was nonadherent, recently relapsed OAA patients. At equal adherence, probability of relapse was equal between PP1M and OAAs, and OAA patients were nonadherent until treatment switch. Event rates (adherence, relapse, and switch) and cost inputs (pharmacy and relapse) were based on the literature, and rates remained constant. Outcomes included number of relapses, pharmacy costs, and relapse costs (2017 U.S. dollars) at years 1,2 , and 3. One-way sensitivity (OSA) and probabilistic sensitivity analyses (PSA) evaluated the effect of varying model inputs on health plan and perpatient level costs.

RESULTS: Based on a hypothetical health plan of 1 million members, 3,037 OAA patients were recently relapsed and nonadherent. Compared with continuing OAAs, switching $5 \%$ of patients $(n=152)$ to PP1M resulted in net cost savings of $\$ 674,975, \$ 723,298$, and $\$ 562,310$ at the plan level; $\$ 4,445, \$ 4,764$, and $\$ 3,703$ per patient switched per year; and $\$ 0.0562$, $\$ 0.0603$, and $\$ 0.0469$ per member per month in years 1,2 , and 3 , respectively, resulting in total plan-level savings of $>\$ 1.9$ million over 3 years. A total of 221 relapses were avoided (year 1: 92; year 2: 72; and year 3: 57). In years 1, 2, and 3, respectively, total annual plan-level schizophreniarelated costs were $\$ 114.1$ million, $\$ 107.2$ million, and $\$ 105.8$ million when all patients switched to PP1M before any subsequent relapse $(n=3,037)$; \$123.4 million, \$109.6 million, and \$106.7 million when patients switched to PP1M after a first subsequent relapse $(n=2,631)$; and $\$ 127.6$ million, $\$ 121.6$ million, and $\$ 117.0$ million when all patients remained on OAAs. The cost per patient switched to PP1M was lower when all patients received PP1M before a subsequent relapse versus after their first subsequent relapse at all years (year $1: \$ 37,559$ vs. $\$ 45,089$; year 2 : $\$ 35,288$ vs. $\$ 36,321$; and year $3: \$ 34,826$ vs. $\$ 35,155)$. OSA demonstrated consistent net cost savings per patient switched, ranging from $\$ 640$ to $\$ 10,484$ (year 1); $\$ 1,774$ to $\$ 9,245$ (year 2); and $\$ 1,354$ to $\$ 7,026$ (year 3). PSA demonstrated $96.3 \%, 99.7 \%$, and $99.7 \%$ of iterations were cost saving in years 1,2 and 3 , respectively.

CONCLUSIONS: Pharmacy costs associated with switching nonadherent OAA patients with a recent relapse to PP1M were offset by reduced relapse rates and health care costs at years 1, 2, and 3, with earlier use of PP1M resulting in increased cost savings at all years.

J Manag Care Spec Pharm. 2020;26(2):176-85

Copyright $\odot 2020$, Academy of Managed Care Pharmacy. All rights reserved.

\section{What is already known about this subject}

Numerous real-world studies have observed enhanced adherence outcomes, as well as decreased rates of relapse and associated schizophrenia-related health care expenditures, among patients treated with paliperidone palmitate (PP1M) versus oral atypical antipsychotics (OAAs).

Previous work used a patient-level clinical trial to project hospitalizations over an 18-month period, demonstrating substantial reductions in psychiatric- and all-cause-related hospitalizations when patients were switched from oral antipsychotics to PP1M or once-every-3-months paliperidone palmitate.

A previous 12-month decision-tree model demonstrated that annual pharmacy costs associated with switching nonadherent Medicaid patients with a recent relapse from OAAs to PP1M were offset by reduced relapse rates and schizophrenia-related health care costs, with earlier use of PP1M projected to generate greater cost savings.

\section{What this study adds}

This study developed a decision tree to investigate the long-term potential health plan effect of transitioning nonadherent and recently hospitalized patients from OAAs to PP1M over a time horizon exceeding 12 months

Extending the previous model to 24 and 36 months showed that pharmacy costs associated with switching nonadherent OAA patients with a recent relapse to PP1M were offset by reduced relapse rates and health care costs at years 1, 2, and 3, with earlier use of PP1M resulting in increased cost savings at all years.

$\square$ chizophrenia, a severe mental illness characterized by a disturbance of thought, perceptions, and emotions, is a common psychotic disorder that affects approximately 20 million individuals worldwide. ${ }^{1,2}$ Early disease onset, along with its chronic trajectory, culminates in a substantial burden on patients, caregivers, payers, and society. ${ }^{3-6}$

Available treatment options for schizophrenia include pharmacological regimens and psychotherapy. Despite the accessibility of treatment, only $20 \%$ of patients report favorable treatment outcomes, with the majority of patients experiencing 
numerous subsequent psychotic episodes, persistent chronic symptoms, and poor treatment continuity. ${ }^{7}$ Suboptimal medication adherence remains a central barrier to effective disease management; in a recent U.S.-based analysis of adult Medicaid patients with schizophrenia, $82.9 \%$ of patients on oral atypical antipsychotics (OAAs) were nonadherent (proportion of days covered $[\mathrm{PDC}]<80 \%$ ) to their antipsychotic regimens. ${ }^{8}$

Over recent years, numerous long-acting injectable (LAI) formulations of antipsychotic medications, such as once-monthly paliperidone palmitate (PP1M), have become available for clinical use. ${ }^{9}$ Such formulations are thought to enhance adherence outcomes due to their long pharmacokinetic half-lives, limited frequency of administration, and increased contact with health care providers. ${ }^{10}$ Current treatment guidelines recommend second-generation long-acting injectable therapies (SGA-LAIs) for the treatment of patients with repeated nonadherence to pharmacological treatment or frequent and recurrent relapse, as well as for the prevention of future nonadherence and relapse. ${ }^{4,11,12}$ The 2017-2018 University of South Florida Best Practice Psychotherapeutic Medication Guidelines for Adults emphasize several additional emerging areas of first-line use for LAIs, highlighting the value of LAIs for use among patient populations who have not been historically referenced. ${ }^{12}$

Despite the vast prevalence of poor treatment adherence, a minority $(<20 \%)$ of patients with schizophrenia are prescribed LAIs, with disproportionately low rates of LAI use reported within the U.S. health care system. ${ }^{9,13-15}$ In a U.S. Medicaid analysis, less than $12 \%$ of patients, a significant proportion of whom were nonadherent, were initiated on SGA-LAIs. ${ }^{16}$ Although underuse of LAIs in the United States may stem from a set of multifaceted issues, high acquisition costs have been cited as a main barrier to their use. ${ }^{9}$

From a U.S. health care system perspective, however, LAIs such as PPIMs have been projected to provide economic benefit across the continuum of psychiatric care due to decreased incidence of relapses or rehospitalizations, which may promote a reduction in costly schizophrenia-related health care expenditures. ${ }^{16-20}$

Numerous real-world studies evaluating PP1M have observed enhanced adherence outcomes and decreased health care costs among patients treated with PP1M compared with OAAs. ${ }^{16-19,21-27}$ This finding has been repeatedly noted among the Medicaid population, ,16,18,19,24,26,27 a health insurance coverage group that has the highest prevalence rate of schizophrenia and serves as the single largest U.S. payer for mental health care services relative to other payer channels. ${ }^{28-30}$ In the Medicaid population, per-patient-per-year all-cause health care costs exceed $\$ 22,000$ among young adults aged 18-25 years with schizophrenia, an age bracket that corresponds with typical disease onset. ${ }^{31,32}$

Notably, 2 recent decision-tree models, leveraging clinical trial and real-world data, demonstrated that pharmacy costs associated with PP1M may be offset by decreased rates of relapse and associated schizophrenia-related health care expenditures., ${ }^{1,33}$ Basu et al. (2018) used PP1M randomized controlled trial data to project the outcome of switching Medicaid patients on oral antipsychotics (OAs) to PP1M; results demonstrated that switching from OAs to PP1M may lead to substantial reductions in psychiatric- and all-cause-related hospitalizations over an 18-month period. ${ }^{33}$ An additional decision-tree model used with a target population of nonadherent Medicaid patients with a recent relapse demonstrated that, over 12 months, pharmacy costs associated with switching patients from OAAs to PPIM were offset by reduced relapse rates and schizophrenia-related health care costs, with earlier use of PPIM (before the incidence of a subsequent relapse) projected to generate greater cost savings. ${ }^{1}$

To our knowledge, no decision-tree model has been developed to investigate the long-term potential health plan effect of transitioning additional nonadherent, recently hospitalized patients from OAAs to PP1M, with "long term" defined as a time period exceeding 18 months. Given PP1M's proven clinical and economic value among nonadherent patients with schizophrenia, it is important to understand if the reduced rates of hospitalization and health care use observed in previous models persist past a 12-month time frame. Such evidence may be leveraged to inform evidence-based decision making among U.S. health care payers and providers in an effort to deliver appropriate care to patients who may derive clinical and economic benefit from PP1M.

This study aimed to extend a previous 12-month decisiontree model to a 24- and 36-month time frame in order to project the long-term economic impact when an incremental proportion of nonadherent patients with a recent relapse switch from OAAs to PP1M.

\section{Methods}

\section{Model Structure and Design}

A 36-month decision-tree model with twelve 3-month cycles was developed from a Medicaid payer's perspective. The long-term model was developed as an extension of a recently published economic model with a 12-month time horizon, ${ }^{1}$ which projected the economic impact when an incremental proportion of nonadherent patients with a recent relapse (defined as a hospitalization within the previous 6 months) were switched from OAAs to PP1M. ${ }^{19}$ At each 3-month cycle, patients could either be adherent or nonadherent to treatment and could experience a subsequent relapse or remain stable. At the end of each cycle, patients could either continue on the same treatment, or switch treatments. The extended model repeats cycles 1-4 (year 1) for cycles 5-12 (years 2 and 3).

A decision-tree model was selected because it has been extensively used in other economic analyses of PP1M and sufficiently captures the patient pathway for individuals with 
schizophrenia in the United States, ${ }^{1,34-38}$ including nonadherent patients and/or patients with a recent relapse episode., ${ }^{1,39}$ Three-month cycles were implemented to be consistent with existing PP1M decision trees, ${ }^{1,34}$ as well as patterns of relapse episodes observed in a claims analysis. ${ }^{40}$ The target population for switching patients to PPIM was nonadherent patients on OAAs with a recent relapse, and the model compared costs (pharmacy and relapse) and frequency of relapse between patients who switched to PP1M and those who remained on OAAs at years 1,2 , and 3 .

To evaluate the economic impact of early treatment with PPIM, the decision-tree analysis considered 3 different scenarios at years 1, 2, and 3: (1) switching 5\% of nonadherent patients with a recent relapse from OAAs to PP1M before they experienced any subsequent relapse; (2) switching 100\% of nonadherent patients with a recent relapse from OAAs to PP1M before they experienced any subsequent relapse; and (3) switching $100 \%$ of nonadherent patients with a recent relapse from OAAs to PPIM immediately after their first subsequent relapse.

\section{Model Assumptions}

The decision-tree model incorporated the following assumptions, which were based on the previous 12 -month model, ${ }^{1}$ and were reviewed by a panel of experts that consisted mainly of U.S. payers (medical and pharmacy directors).

1. The target population for switching patients to PP1M was nonadherent patients on OAAs who had at least 1 recent relapse.

2. At equal adherence, the risk of relapse was equal between PP1M and OAAs; a conservative assumption was made due to limited literature regarding relapse risk among patients on PP1M or OAAs with similar rates of adherence.

3. Patients transitioned through twelve 3-month cycles over a 36-month time frame and could experience up to 12 relapses ( 1 per cycle); this pattern is consistent with a mean duration between relapse episodes of 12.5 weeks, as observed in a claims analysis. ${ }^{40}$

4. Pharmacy costs were calculated for each 3-month cycle.

5. Patients could switch treatments multiple times; however, those on PPIM could only switch to an OAA, and those on OAAs could only switch to another OAA.

6. Nonadherent patients on OAAs who switched to another OAA could be adherent to their new OAA treatment and were no longer assumed to be nonadherent only.

7. In the scenario where $5 \%$ of patients were switched from OAAs to PP1M before any subsequent relapse, patients switching to PP1M were assumed to be part of the PP1M arm regardless of a switch to OAAs over the course of the model; in the scenario where patients were switched from OAAs to PP1M at a specified number of subsequent relapses, all patients on OAAs who experienced a subsequent relapse were switched to PPIM at the next cycle.

\section{Model Inputs}

Model inputs were derived from a targeted literature search that focused on publications that evaluated a large population of adults with schizophrenia treated with OAAs or SGA-LAIs (specifically PP1M), were published within the last 5 years, and were conducted from a U.S. payer's perspective.

Model inputs were the same as those used in the previous model. ${ }^{1}$ Inputs remained constant over 36 months in order to observe the continuous long-term implications of switching patients from OAAs to PPIM without introducing confounding changes to the model framework that have not been explicitly justified by the literature.

Population Inputs. The target population for switching patients to PP1M was nonadherent patients on OAAs with a recent relapse, as consistent with the previous model. ${ }^{1}$ A specific population of nonadherent patients with a recent relapse was selected in order to more realistically evaluate the patient pathway for the subset of patients for whom PP1M is strongly recommended by the treatment guidelines specifying LAI use., ${ }^{4,12}$ This target population may also derive the greatest incremental benefit from a treatment switch to PP1M., ${ }^{4,12,39,41}$ Population inputs used in the model are summarized in El Khoury et al. (2019). ${ }^{1}$

Clinical Event Probabilities. All clinical event probabilities mirrored the previous model and were adjusted to 3-month cycles. ${ }^{1}$ Adherence was defined as $\mathrm{PDC} \geq 80 \%$. The probability of relapse was 0.261 for adherent patients and 0.492 for nonadherent patients, based on rehospitalization rates from Lafeuille et al. (2013), adherence rates from Marcus et al. (2015), and a nonadherent/adherent ratio of rehospitalization of 1.887 from Ascher-Svanum et al. (2006). 17,19,42 Probability of adherence was consistent across all cycles unless a patient switched from PP1M to an OAA or from an OAA to a different OAA, and probability of relapse was consistent across all cycles and treatment unless a patient's adherence status changed. At each cycle, patients were subject to nonadherence or adherence, regardless of their adherence status in the previous cycle. Probabilities of adherence for PPIM (0.714) and OAA (0.568) remained constant each year and consistent with published literature, suggesting that cross-sectional levels of adherence remain relatively stable after the first 6 or 12 months of treatment with antipsychotics, although patient-level probabilities of adherence may fluctuate over time..$^{19,42-44}$

Because of limited data on probability of treatment switch among nonadherent or adherent patients with or without a previous relapse, the probability of switch inputs were largely informed by clinical assumptions. Although real-world data describing switch rates based on adherence levels are sparse, 
available evidence suggests that $18 \%$ of patients on PP1M switched to an OAA during an average observation period of 10.9 months. ${ }^{10}$ Consequently, assuming a $20 \%$ probability of switching antipsychotic regimens among stable patients was considered a fair estimate. Clinical event probabilities are detailed in El Khoury et al. ${ }^{1}$

Cost Inputs. All costs were consistent with the 12-month model. ${ }^{1}$ Pharmacy costs were calculated for each 3-month cycle. Cost of PP1M was derived from RED BOOK Online and based on the dosing recommendations as per the U.S. Food and Drug Administration (FDA) prescribing information. ${ }^{45,46}$ A $23 \%$ discount rate was applied to the cost of PP1M for consistency with the statutory discount for the Medicaid drug rebate program. ${ }^{47}$ The first month PP1M pharmacy cost (calculated as initiation dose of $234 \mathrm{mg}$ + additional administration dose of $156 \mathrm{mg}$ ) was $\$ 4,170$, discounted to $\$ 3,211$. Regular maintenance dose of PP1M (117 mg) was $\$ 1,251$, discounted to $\$ 963$.

The average monthly cost of OAAs (\$341) was calculated as a weighted cost of an OAA case mix that included aripiprazole, asenapine maleate, iloperidone, lurasidone, olanzapine, paliperidone, quetiapine, risperidone, and ziprasidone. Weighted cost was determined based on the average wholesale acquisition cost obtained from RED BOOK Online and the recommended dosing based on the American Psychiatric Association practice guidelines (where available) or as per FDA prescribing information. ${ }^{4,45,48-51}$ Each agent was weighted to contribute to the OAA case mix based on medication utilization frequencies reported in Pilon et al. (2017). ${ }^{52}$

The model included an average cost per relapse episode of $\$ 20,995$, containing the following components: (a) average cost for an initial inpatient stay of $\$ 9,693$, based on a weighted average of mean costs for schizophrenia-related hospitalizations (International Classification of Diseases, Ninth Revision, Clinical Modification codes 295.xx) from the Healthcare Cost and Utilization Project (HCUP) ${ }^{53}$; (b) average cost of $\$ 1,832$ for a schizophrenia-related readmission from HCUP (18.6\% of patients were readmitted within 30 days at a cost of $\$ 8,600)^{54}$; and (c) average estimated cost for outpatient and other medical ancillary costs of $\$ 9,470$, using a ratio of $43.3 \%$ to $52.7 \%$ for outpatient and other medical ancillary costs relative to institutional costs from Lafeuille et al. (2013). ${ }^{40}$

Costs were standardized using the Consumer Price Index to 2017 U.S. dollars.

\section{Statistical Analysis}

Statistical analyses were consistent with the previous 12-month model. ${ }^{1} \mathrm{~A}$ total of 3,037 OAA nonadherent patients with a recent relapse were included in the decision tree. ${ }^{1}$ At each of the twelve 3-month cycles, patients were able to be adherent or nonadherent to their treatments, experience relapses, and/or switch antipsychotic medications. Clinical event probabilities were conditional on the patients' previous events; the probability of relapse was dependent on previous adherence, and the probability of switching treatment was dependent on previous adherence and relapse.

A one-way sensitivity analysis (OSA) evaluated the impact of varying model inputs on health plan and per-patient costs. Probabilities of relapse and switch for the OSA were obtained from published literature to create a range of clinical event rates with upper and lower bounds. ${ }^{1}$ Because probabilities of adherence used in the model were conservative and consistent with the lower bounds identified in the targeted literature review, the OSA applied $\pm 30 \%$ to rates of adherence and relapse costs. Treatment costs were varied by $\pm 20 \%$, and the impact of a generic OAA acquisition cost of $\$ 50$ was assessed. An additional OSA evaluated the impact of varying dosage strength and associated acquisition costs of PP1M based on treatment patterns reflecting real-world considerations. ${ }^{55}$

A probabilistic sensitivity analysis (PSA) evaluated the impact of varying all model inputs using a Monte Carlo simulation with 1,000 iterations. ${ }^{1}$ Model inputs were sampled at random from the upper and lower bounds identified in the OSA. Clinical event rates, pharmacy costs, and relapse costs were assumed to follow a uniform probability distribution in order to apply equal likelihood to the range of inputs identified through the targeted literature or selected as part of the sensitivity analyses.

Analyses were conducted using Microsoft Excel version 2016 (Microsoft, Redmond, WA).

\section{Results}

\section{Switching a Fixed Proportion (5\%) of Patients to PP1M Before Any Subsequent Relapse}

Compared with continuing treatment with OAAs, switching $5 \%$ of recently relapsed, nonadherent patients ( $\mathrm{n}=152)$ from OAAs to PP1M before any subsequent relapse resulted in net schizophrenia-related cost savings at the plan and patient level (Table 1). ${ }^{1}$ This switching scenario was associated with total plan-level savings of \$1,960,583 across all 3 years, largely driven by a reduction in health plan-level relapse costs $(-\$ 4,628,214)$, which offset the increased plan-level pharmacy costs $(\$ 2,667,632)$ associated with PP1M.

Switching 5\% of patients from OAAs to PPlM before any subsequent relapse averted a total of 221 relapses over 3 years. ${ }^{1}$ The proportion of patients who were relapse-free each given year increased slightly over time (year 1: $7.6 \%$, year 2 : $9.6 \%$, and year 3: 11.1\%).

\section{Switching All Patients to PP1M Before and After a Subsequent Relapse}

In years 1,2 , and 3, respectively, total annual plan-level schizophrenia-related costs were $\$ 114.1, \$ 107.2$, and $\$ 105.8$ million when all patients switched to PP1M before any subsequent relapse ( $\mathrm{n}=3,037) ; \$ 123.4, \$ 109.6$, and $\$ 106.7$ million 


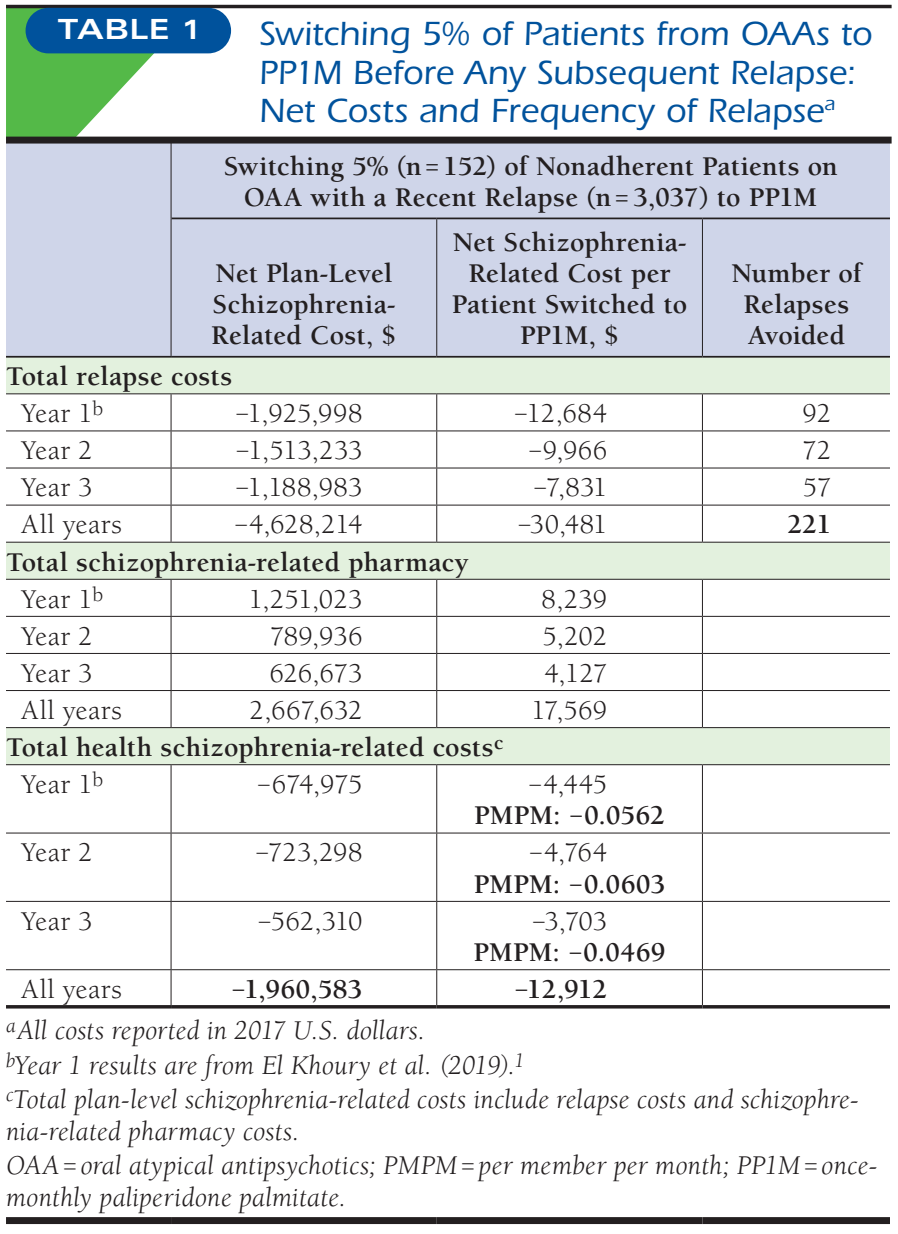

when patients switched to PP1M after a first subsequent relapse $(n=2,631)$; and $\$ 127.6, \$ 121.6$, and $\$ 117.0$ million when all patients remained on OAAs (Table 2). ${ }^{1}$ The cost per patient switched to PP1M was lower when all patients received PP1M before a subsequent relapse versus after their first subsequent relapse at all years (year 1: $\$ 37,559$ vs. $\$ 45,089$; year 2 : $\$ 35,288$ vs. $\$ 36,321$; and year 3 : $\$ 34,826$ vs. $\$ 35,155)$.

In years 1,2 , and 3 , respectively, the total number of relapses was 5,834, 5,517, and 5,270 when all patients stayed on OAAs; 3,999, 4,076, and 4,137 when all patients switched to PPIM on the first cycle before any subsequent relapse; and $4,916,4,088$, and 4,104 when all patients switched to PP1M after their first subsequent relapse. ${ }^{1}$ Across all years, the number of relapses associated with switching all patients before a subsequent relapse was consistently lower than the number of relapses associated with switching patients after a subsequent relapse or continuing OAAs.

By 3 years, switching patients to PP1M before any subsequent relapse averted 896 relapses (12,212 vs. 13,108), when compared with switching patients after a subsequent relapse, and 4,409 relapses $(12,212$ vs. 16,621$)$ when compared with continuing all patients on OAAs (Table 1).

\section{One-Way Sensitivity Analysis}

After varying all model inputs in the OSA, Figure 1 highlights the input parameters that influenced the schizophrenia-related net cost per patient switched from OAAs to PP1M, when 5\% of all patients were switched before any subsequent relapse at year 2 and year 3; OSA results for year 1 are presented in El Khoury et al. ${ }^{1}$

Exploratory OSA demonstrated that net cost savings associated with PP1M were robust across a range of scenarios, including variations in probability of relapse, probability of adherence, probability of switch, and pharmacy costs. Irrespective of model inputs, PP1M consistently demonstrated a net cost savings in years 1-3. ${ }^{1}$ The net cost savings per patient switched to PPlM ranged from $\$ 640$ to $\$ 10,484$ in year $1,{ }^{1} \$ 1,774$ to $\$ 9,245$ in year 2 , and $\$ 1,354$ to $\$ 7,026$ in year 3 . The OSA demonstrated that the net cost per patient switched from OAAs to PP1M was most sensitive to the relapse ratio (probability of relapse among nonadherent patients divided by probability of relapse among adherent patients), followed by the relapse cost. In year 1 , probability of relapse was the third most important driver $^{1}$; in years 2 and 3 , probability of adherence emerged as the third most important driver.

An additional OSA varied dosing patterns and associated acquisition costs of PP1M (based on treatment patterns in Pilon et al., 2016 $6^{56}$ ) in all years for all switching scenarios; results were insensitive. ${ }^{1}$ Savings were also consistently observed when a generic OAA acquisition cost of $\$ 50$ (vs. $\$ 341$ in the base case) was used; in this scenario, net cost savings per patient switched were $\$ 3,208, \$ 3,769$, and $\$ 2,900$ in years 1 , 2 , and 3 , respectively.

\section{Probabilistic Sensitivity Analysis}

After varying all model inputs simultaneously in the PSA, the figure in the Appendix (available in online article) demonstrates the distribution of schizophrenia-related net cost per patient switched from OAAs in years 2 and 3; PSA results for year 1 are presented in El Khoury et al..$^{1}$ The cost advantage associated with PP1M decreased slightly over time, with year 1 demonstrating the greatest net cost savings; however, there was a cost advantage associated with PP1M at all 3 years. In year 1 , mean cost savings across the 1,000 iterations was $\$ 5,642$ per patient, with an interquartile range (IQR) of $\$ 4,885(\$ 2,974$ $\$ 7,859)$; of the 1,000 iterations, $96.3 \%$ demonstrated a net cost savings per patient switched to PP1M. In year 2, mean net cost savings across the 1,000 iterations was $\$ 5,250$ per patient, with an IQR of $\$ 3,736(\$ 3,068-\$ 6,804)$; of the 1,000 iterations, $99.7 \%$ demonstrated a net cost savings per patient switched to PP1M. Cumulatively, as of year 2 (including years 1-2), 


\begin{tabular}{|c|c|c|c|}
\hline TABLE 2 & \multicolumn{3}{|c|}{$\begin{array}{l}\text { Switching } 100 \% \text { of Patients from OAAs } \\
\text { to PP1M Before Any Subsequent Relapse } \\
\text { and After a First Subsequent Relapse: } \\
\text { Costs and Frequency of Relapse }\end{array}$} \\
\hline & $\begin{array}{l}\text { All Patients } \\
\text { Staying on } \\
\text { OAAs }\end{array}$ & $\begin{array}{l}\text { Switching All } \\
\text { Patients to } \\
\text { PPIM Before } \\
\text { Any Subsequent } \\
\text { Relapse } \\
\end{array}$ & $\begin{array}{l}\text { Switching } \\
\text { Patients to PPIM } \\
\text { After a First } \\
\text { Subsequent } \\
\text { Relapse } \\
\end{array}$ \\
\hline $\begin{array}{l}\text { Patients switching } \\
\text { to PP1M, n (\%) }\end{array}$ & $0(0.0)$ & $3,037(100.0)$ & $2,631(86.6)$ \\
\hline \multicolumn{4}{|c|}{ Plan-level schizophrenia-related costs, \$ } \\
\hline \multicolumn{4}{|c|}{ Relapse } \\
\hline Year $1^{b}$ & $122,483,568$ & $83,963,606$ & $103,217,990$ \\
\hline Year 2 & $115,839,751$ & $85,575,082$ & $85,821,198$ \\
\hline Year 3 & $110,637,571$ & $86,857,916$ & $86,157,698$ \\
\hline All years & $348,960,890$ & $256,396,604$ & $275,196,887$ \\
\hline \multicolumn{4}{|l|}{ Pharmacy } \\
\hline Year $1^{\mathrm{b}}$ & $5,075,602$ & $30,096,069$ & $20,166,864$ \\
\hline Year 2 & $5,800,079$ & $21,587,258$ & $23,786,878$ \\
\hline Year 3 & $6,367,353$ & $18,900,807$ & $20,555,270$ \\
\hline All years & $17,243,033$ & $70,584,134$ & $64,509,013$ \\
\hline \multicolumn{4}{|l|}{ Total } \\
\hline Year $1^{b}$ & $127,559,170$ & $114,059,675$ & $123,384,855$ \\
\hline Year 2 & $121,639,830$ & $107,162,340$ & $109,608,076$ \\
\hline Year 3 & $117,004,923$ & $105,758,723$ & $106,712,969$ \\
\hline All years & $366,203,923$ & $326,980,738$ & $339,705,900$ \\
\hline \multicolumn{4}{|c|}{ Schizophrenia-related cost per patient switched to PPIM, \$ } \\
\hline \multicolumn{4}{|c|}{ Relapse } \\
\hline Year $1^{\mathrm{b}}$ & - & 27,649 & 37,674 \\
\hline Year 2 & - & 28,180 & 28,430 \\
\hline Year 3 & - & 28,602 & 28,383 \\
\hline \multicolumn{4}{|l|}{ Pharmacy } \\
\hline Year $1^{b}$ & - & 9,911 & 7,416 \\
\hline Year 2 & - & 7,109 & 7,891 \\
\hline Year 3 & - & 6,224 & 6,772 \\
\hline \multicolumn{4}{|l|}{ Total } \\
\hline Year $1^{\mathrm{b}}$ & - & 37,559 & 45,089 \\
\hline Year 2 & - & 35,288 & 36,321 \\
\hline Year 3 & - & 34,826 & 35,155 \\
\hline \multicolumn{4}{|c|}{ Number of relapses } \\
\hline Year $1^{\mathrm{b}}$ & 5,834 & 3,999 & 4,916 \\
\hline Year 2 & 5,517 & 4,076 & 4,088 \\
\hline Year 3 & 5,270 & 4,137 & 4,104 \\
\hline All years & 16,621 & 12,212 & 13,108 \\
\hline \multicolumn{4}{|c|}{$\begin{array}{l}\text { aAll costs reported in } 2017 \text { U.S. dollars. } \\
\text { bYear } 1 \text { results are from El Khoury et al. }(2019) .{ }^{1} \\
\text { OAA= oral atypical antipsychotics; } P P 1 M=\text { once-monthly paliperidone palmitate. }\end{array}$} \\
\hline
\end{tabular}

99.9\% of iterations demonstrated a net cost savings per patient switched. In year 3, net cost savings across the 1,000 iterations was $\$ 3,853$ per patient, with an IQR of $\$ 3,051$ ( $\$ 2,005-\$ 5,056)$; of the 1,000 iterations, $99.7 \%$ demonstrated a net cost savings per patient switched to PP1M. Cumulatively, as of year 3 (including years 1-3), 100\% of iterations demonstrated a net cost savings per patient switched.

\section{Discussion}

Over 36 months, pharmacy costs associated with switching nonadherent Medicaid patients on OAAs with a recent relapse to PP1M were fully offset by reduced rates of relapse and associated health care expenditures, leading to net cost savings at the patient and health plan levels at years 1,2 , and $3 .{ }^{1}$ Hence, the long-term benefit associated with switching nonadherent patients with a recent relapse from OAAs to PPlM persisted over time, suggesting a long-term economic value for PP1M among recently relapsed, nonadherent patients when assuming constant clinical event probabilities in years 1-3. Results were robust across both OSA and PSA in years 1-3, with modifications to the relapse ratio, relapse cost, and probability of relapse demonstrating the greatest impact on model results. Cost savings were consistently observed when considering varied dosing patterns and associated costs of PP1M and a generic OAA acquisition cost of $\$ 50$. Although cost savings were greatest in year 1 , savings were demonstrated consistently by the PSA for all years. ${ }^{1}$

Additionally, earlier treatment with PP1M, before the incidence of a subsequent relapse, was projected to generate greater cost savings for all 3 years, evident by the results of switching all patients from OAAs to PP1M at the first cycle before any subsequent relapse as opposed to after a subsequent relapse. ${ }^{1}$

Results of this study have numerous real-world implications, relevant to health care providers and payers. From an economic perspective, results suggest that treating nonadherent, relapsing patients who drive high health care costs with PP1M relative to OAAs may be a long-term cost-saving strategy for U.S. health plans, ${ }^{56}$ specifically for Medicaid patients, a health care plan coverage group with the largest estimated prevalence of schizophrenia. ${ }^{28,30}$ Of note, the current analysis accounted for the statutory rebates required under the Medicaid Drug Rebate Program, ${ }^{4,12,47}$ which distinguishes the model from previous economic analyses of PP1M.

As of 2020, patients who demonstrate acceptable adherence to their antipsychotic regimens still may not meet criteria for insurance reimbursement of LAIs. A set of roundtable experts have recommended that patients should not have to first prove that they have relapsed on OAs in order to secure favorable reimbursement for LAIs. ${ }^{9}$ In this analysis, when all patients were switched to PP1M before any subsequent relapse, 20.5\% of patients were relapse-free within the first year, which was considerably greater than the proportion of patients relapsefree for the first year in the scenario when all patients were switched after experiencing a first relapse (6.9\%). These results underscore recommendations of the roundtable experts, substantiating the benefit of switching patients to PPlM even if they have not experienced a subsequent relapse. ${ }^{9}$ Considering that relapse evokes detrimental consequences at multiple clinical, societal, and financial dimensions, preventing the likelihood of future relapses should be an important goal of the U.S. 
Projecting the Long-Term Economic Impact of Once-Monthly Paliperidone

Palmitate Versus Oral Atypical Antipsychotics in Medicaid Patients with Schizophrenia

FIGURE 1 Sensitivity Analysis: Net Schizophrenia-Related Cost of Switching an OAA Nonadherent Patient with a Recent Relapse to PP1M at Years 2 and $3^{a}$

Year 2

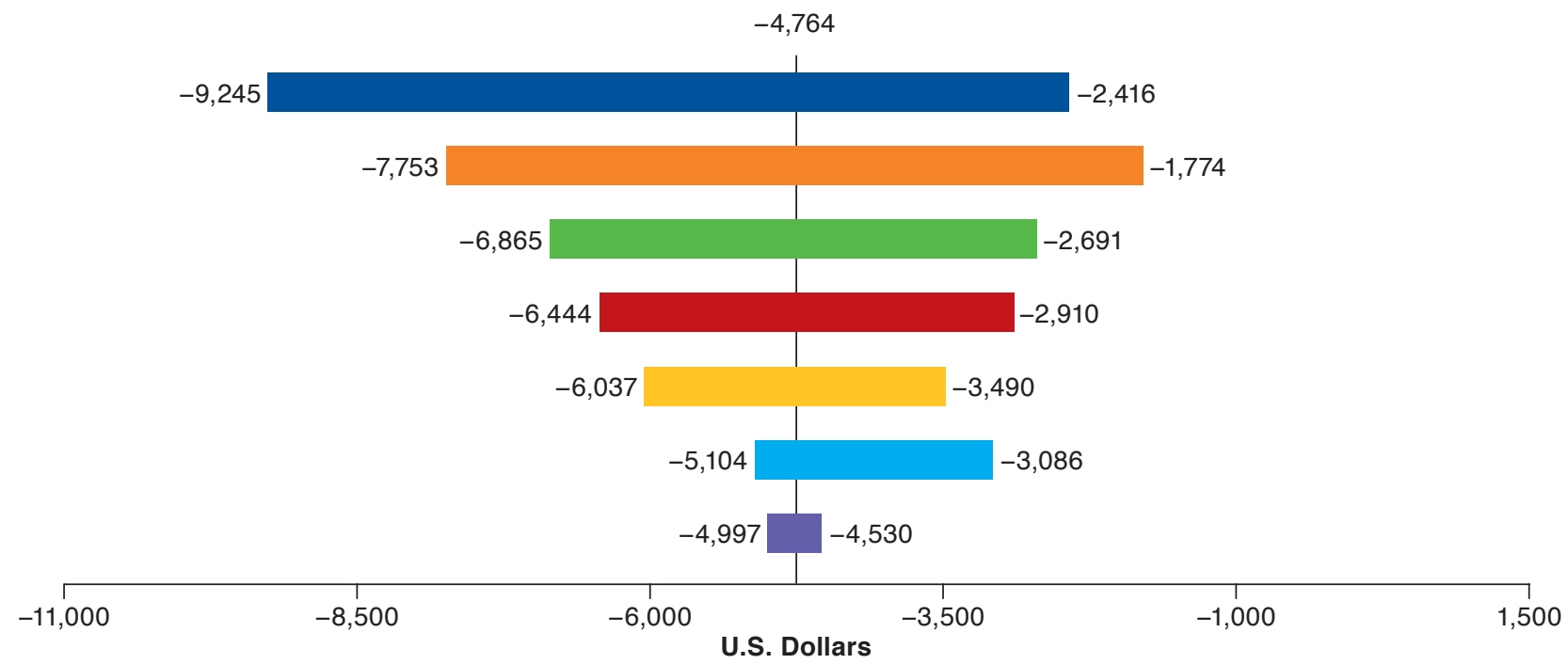

Year 3

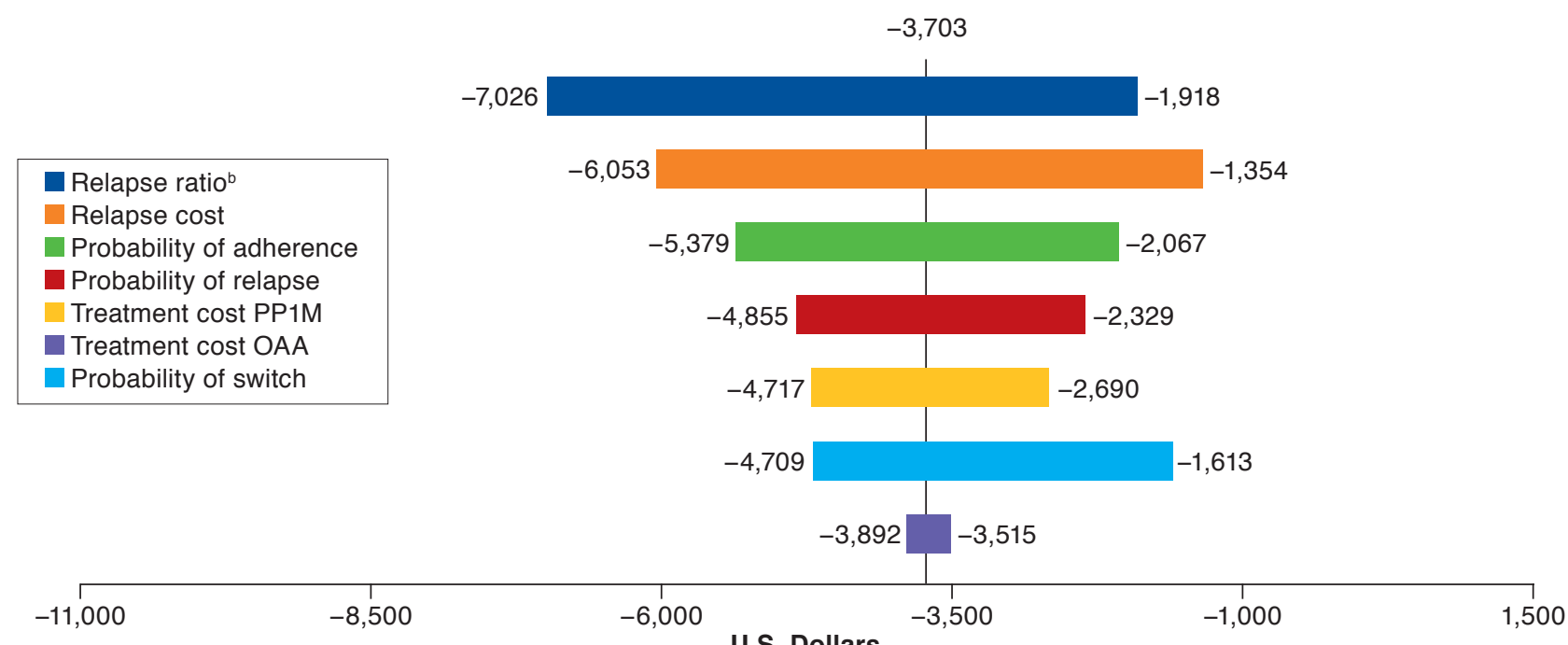

Note: Upper and lower bounds for each input used in the OSA are as follows: Probability of adherence, PP1M (0.4999, 0.9284); Probability of adherence, OAA (0.3978, $0.7388)$; Probability of rehospitalization for the relapse ratio, adherent $(0.2165,0.2828)$; Probability of rehospitalization for the relapse ratio, nonadherent $(0.4570$, 0.5596); Probability of relapse, adherent (0.2084, 0.3126); Probability of relapse, nonadherent (0.3932, 0.5897); Probability of switch (0.0200, 0.1500); Cost of relapse $(\$ 14,697, \$ 27,294$; Cost of PP1M monthly (\$771, \$1,156); Cost of OAA monthly (\$273, \$409).

aAll costs reported in 2017 U.S. dollars.

${ }^{b}$ Defined as the probability of rehospitalization among nonadherent patients/probability of rehospitalization among adherent patients.

$O A A=$ oral atypical antipsychotics; $O S A=$ one-way sensitivity analysis; $P P 1 M=$ once-monthly paliperidone palmitate.

health care system, even for those patients who may not have a documented history of relapse or nonadherence. Additionally, health care decision makers should encourage practices that improve long-term patient and population outcomes while reducing health care expenditures (i.e., academic detailing, decision support, clinical pathways, and case management).

From a clinician's perspective, model outcomes were consistent with current treatment guidelines, suggesting that LAIs 
may be considered as an early treatment for a heterogeneous set of patients, providing individuals at all stages of disease with an increased likelihood of symptomatic remission. ${ }^{1}$

\section{Limitations}

This study has a number of limitations. The target population only included Medicaid patients with schizophrenia that were nonadherent with a recent relapse switching to PP1M, so the results of the model may not be generalizable to the overall, heterogeneous population of individuals with schizophrenia, representative of alternative U.S. payer channels, or generalizable to all LAIs. However, given the high prevalence of nonadherence and relapse in schizophrenia, ${ }^{30}$ these results provide acceptable basis for evidence-based medical policies.

In addition, clinical event rates and cost inputs were limited to data from the literature. Literature regarding switch rates among patients who are nonadherent versus adherent was sparse, although data suggest that our estimates were reasonable. ${ }^{10}$ Furthermore, costs from HCUP may be underestimates, since they do not include professional fees, and adverse event costs were not incorporated into the cost model calculation. ${ }^{53}$

Finally, the hypothetical population considered prevalence estimates for the general U.S. population, thereby potentially understating the prevalence of schizophrenia among Medicaid beneficiaries and providing a conservative estimate of net schizophrenia-related cost savings. ${ }^{1}$

\section{Conclusions}

$\overline{\text { Based on the analysis of switching nonadherent OAA patients }}$ with a recent relapse to PP1M, pharmacy costs were offset by reduced relapse rates and health care costs at years 1,2 , and 3 , with earlier use of PP1M resulting in increased cost savings at all years. ${ }^{1}$

\section{Authors}

ANTOINE C. EL KHOURY, PhD, and EDWARD KIM, MD, MBA, Janssen Scientific Affairs, Titusville, New Jersey. DOMINIC PILON, MA; LAURA MORRISON, MSc; and PATRICK LEFEBVRE, MA, Analysis Group, Montréal, Quebec, Canada. NINA SHAK, BA, Analysis Group, Menlo Park, California, and AMANDA LLANEZA, BA, HireGenics, Duluth, Georgia.

AUTHOR CORRESPONDENCE: Antoine C. El Khoury, PhD, Janssen Scientific Affairs, 1125 Trenton Harbourton Rd., Titusville, NJ 08560.E-mail: AKhour@its.jnj.com.

\section{DISCLOSURES}

This research was funded by Janssen Scientific Affairs. Pilon, Morrison, Lefebvre, and Shak are employees of Analysis Group, a consulting company that received research grants from Janssen Scientific Affairs to conduct this study. El Khoury and Kim are employees of Janssen Scientific Affairs. At the time this study was conducted, Llaneza was an employee of HireGenics, which provided services to Janssen Scientific Affairs for the study.

Part of the material in this manuscript was presented at the Academy of Managed Care Pharmacy 2019 Annual Meeting; March 25-29, 2019; San Diego, CA.

\section{REFERENCES}

1. El Khoury AC, Pilon D, Morrison L, et al. The prospective economic impact of once-monthly paliperidone palmitate versus oral atypical antipsychotics in Medicaid patients with schizophrenia. Curr Med Res Opin. 2019;35(3):395-405.

2. World Health Organization. Schizophrenia. October 4, 2019. Available at: https://www.who.int/news-room/fact-sheets/detail/schizophrenia. Accessed January 17, 2020.

3. Emsley R, Chiliza B, Asmal L, Harvey BH. The nature of relapse in schizophrenia. BMC Psychiatry. 2013;13:50.

4. Lehman AF, Lieberman JA, Dixon LB, et al. Practice guideline for the treatment of patients with schizophrenia, second edition. Am J Psychiatry. 2004;161(2 Suppl):1-56.

5. Gopal S, Xu H, McQuarrie K, et al. Caregiver burden in schizophrenia following paliperidone palmitate long acting injectables treatment: pooled analysis of two double-blind randomized phase three studies. NPJ Schizophren. 2017;3(1):23.

6. Wu EQ, Birnbaum HG, Shi L, et al. The economic burden of schizophrenia in the United States in 2002. J Clin Psychiatry. 2005;66(9):1122-29.

7. Patel KR, Cherian J, Gohil K, Atkinson D. Schizophrenia: overview and treatment options. PT. 2014;39(9):638-45.

8. Manjelievskaia J, Amos TB, El Khoury AC, Vlahiotis A, Cole A, Juneau P. A comparison of treatment patterns, healthcare resource utilization, and costs among young adult Medicaid beneficiaries with schizophrenia treated with paliperidone palmitate or oral atypical antipsychotics in the U.S. J Med Econ. 2018;21(12):1221-29.

9. Correll CU, Citrome L, Haddad PM, et al. The use of long-acting injectable antipsychotics in schizophrenia: evaluating the evidence. J Clin Psychiatry. 2016;77(Suppl 3):1-24.

10. Xiao Y, Muser E, Lafeuille MH, et al. Impact of paliperidone palmitate versus oral atypical antipsychotics on healthcare outcomes in schizophrenia patients. J Comp Eff Res. 2015;4(6):579-92.

11. Kane JM, Garcia-Ribera C. Clinical guideline recommendations for antipsychotic long-acting injections. Br J Psychiatry Suppl. 2009;195(52):S63-S67.

12. University of South Florida, Florida Medicaid Drug Therapy Management Program for Behavioral Health, Florida Agency for Health Care Administration. 2017-2018 Florida best practice psychotherapeutic medication guidelines for adults. 2018. Available at: https://medicaidmentalhealth.fmhi.usf.edu/_assets/file/Guidelines/2018-Psychotherapeutic\%20 Medication\%20Guidelines\%20for\%20Adults\%20with\%20References.pdf. Accessed December 27, 2019.

13. Fayek M, Flowers C, Signorelli D, Simpson G. Psychopharmacology: underuse of evidence-based treatments in psychiatry. Psychiatr Serv. 2003;54(11):1453-54, 1456.

14. Kaplan G, Casoy J, Zummo J. Impact of long-acting injectable antipsychotics on medication adherence and clinical, functional, and economic outcomes of schizophrenia. Patient Prefer Adherence. 2013;7:1171-80.

15. Kishimoto T, Sanghani S, Russ MJ, et al. Indications for and use of longacting injectable antipsychotics: consideration from an inpatient setting. Int Clin Psychopharmacol. 2017;32(3):161-68. 
16. Pilon D, Tandon N, Lafeuille MH, et al. Treatment patterns, health care resource utilization, and spending in Medicaid beneficiaries initiating second-generation long-acting injectable agents versus oral atypical antipsychotics. Clin Ther. 2017;39(10):1972-85.e2.

17. Lafeuille M-H, Laliberté-Auger F, Lefebvre P, Frois C, Fastenau J, Duh MS. Impact of atypical long-acting injectable versus oral antipsychotics on rehospitalization rates and emergency room visits among relapsed schizophrenia patients: a retrospective database analysis. BMC Psychiatry. 2013;13(1):221.

18. Pilon D, Muser E, Lefebvre P, Kamstra R, Emond B, Joshi K. Adherence, healthcare resource utilization and Medicaid spending associated with oncemonthly paliperidone palmitate versus oral atypical antipsychotic treatment among adults recently diagnosed with schizophrenia. BMC Psychiatry. 2017;17(1):207.

19. Marcus SC, Zummo J, Pettit AR, Stoddard J, Doshi JA. Antipsychotic adherence and rehospitalization in schizophrenia patients receiving oral versus long-acting injectable antipsychotics following hospital discharge. J Manag Care Spec Pharm. 2015;21(9):754-68. Available at: https://www.jmcp. org/doi/10.18553/jmcp.2015.21.9.754

20. Offord S, Wong B, Mirski D, Baker RA, Lin J. Healthcare resource usage of schizophrenia patients initiating long-acting injectable antipsychotics vs. oral. J Med Econ. 2013;16(2):231-39.

21. Lafeuille MH, Grittner AM, Fortier J, et al. Comparison of rehospitalization rates and associated costs among patients with schizophrenia receiving paliperidone palmitate or oral antipsychotics. Am J Health Syst Pharm. 2015;72(5):378-89.

22. Young-Xu Y, Duh MS, Muser E, et al. Impact of paliperidone palmitate versus oral atypical antipsychotics on health care resource use and costs in veterans with schizophrenia. J Clin Psychiatry. 2016;77(10):e1332-e1341.

23. Lefebvre P, Muser E, Joshi K, et al. Impact of paliperidone palmitate versus oral atypical antipsychotics on health care resource use and costs in veterans with schizophrenia and comorbid substance abuse. Clin Ther. 2017;39(7):1380-95.e4.

24. Joshi K, Lafeuille MH, Kamstra R, et al. Real-world adherence and economic outcomes associated with paliperidone palmitate versus oral atypical antipsychotics in schizophrenia patients with substance-related disorders using Medicaid benefits. J Comp Eff Res. 2018;7(2):121-33.

25. Baser O, Xie L, Pesa J, Durkin M. Healthcare utilization and costs of Veterans Health Administration patients with schizophrenia treated with paliperidone palmitate long-acting injection or oral atypical antipsychotics. $J$ Med Econ. 2015;18(5):357-65.

26. Pesa JA, Muser E, Montejano LB, Smith DM, Meyers OI. Costs and resource utilization among Medicaid patients with schizophrenia treated with paliperidone palmitate or oral atypical antipsychotics. Drugs Real World Outcomes. 2015;2(4):377-85

27. Pesa JA, Doshi D, Wang L, Yuce H, Baser O. Health care resource utilization and costs of California Medicaid patients with schizophrenia treated with paliperidone palmitate once monthly or atypical oral antipsychotic treatment. Curr Med Res and Opin. 2017;33(4):723-31.

28. Khaykin E, Eaton WW, Ford DE, Anthony CB, Daumit GL. Health insurance coverage among persons with schizophrenia in the United States. Psychiatr Serv. 2010;61(8):830-834.

29. Centers for Medicare and Medicaid Services. Behavioral health services. 2019. Available at: https://www.medicaid.gov/medicaid/benefits/bhs/index. html. Accessed December 27, 2019.

30. Wu EQ, Shi L, Birnbaum H, Hudson T, Kessler R. Annual prevalence of diagnosed schizophrenia in the USA: a claims data analysis approach. Psychological Medicine. 2006;36(11):1535-40.

31. National Alliance on Mental Illness. Schizophrenia. 2019. Available at: https://www.nami.org/learn-more/mental-health-conditions/schizophrenia. Accessed December 27, 2019.

32. Huang A, Amos TB, Joshi K, Wang L, Nash A. Understanding healthcare burden and treatment patterns among young adults with schizophrenia. J Med Econ. 2018;21(10):1026-35.
33. Basu A, Benson C, Alphs L. Projecting the potential effect of using paliperidone palmitate once-monthly and once-every-3-months long-acting injections among Medicaid beneficiaries with schizophrenia. J Manag Care Spec Pharm. 2018;24(8):759-68. Available at: https://www.jmcp.org/ doi/10.18553/jmcp.2018.24.8.759.

34. Nuhoho S, Saad A, Saumell G, Ribes D, El Khoury AC. Economic evaluation of paliperidone palmitate once monthly for treating chronic schizophrenia patients in the United Arab Emirates. Cur Med Res Opin. 2018;34(4):601-11.

35. Einarson TR, Maia-Lopes S, Goswami P, Bereza BG, Van Impe K. Economic analysis of paliperidone long-acting injectable for chronic schizophrenia in Portugal. J Med Econ. 2016;19(9):913-21.

36. Hemels M, Einarson TR, Zilbershtein R, Schubert A, SkrzekowskaBaran I, van Impe K. Cost-effectiveness of injectable atypical longacting antipsychotics for chronic schizophrenia in Poland. Value Health. 2013;16(7):A548 [abstract]. Available at: https://www.valueinhealthjournal com/article/S1098-3015(13)03311-1/fulltext. Accessed December 27, 2019.

37. Einarson TR, Vicente C, Zilbershtein R, et al. Pharmacoeconomics of depot antipsychotics for treating chronic schizophrenia in Sweden. Nord J Psychiatry. 2014;68(6):416-27.

38. Einarson TR, Geitona M, Chaidemenos A, et al. Pharmacoeconomic analysis of paliperidone palmitate for treating schizophrenia in Greece. Ann Gen Psychiatry. 2012;11(1):18.

39. Einarson TR, Pudas H, Goswami P, van Impe K, Bereza BG. Pharmacoeconomics of long-acting atypical antipsychotics for acutely relapsed chronic schizophrenia in Finland. J Med Econ. 2016;19(2):111-20.

40. Lafeuille M-H, Gravel J, Lefebvre P, et al. Patterns of relapse and associated cost burden in schizophrenia patients receiving atypical antipsychotics. J Med Econ. 2013;16(11):1290-99.

41. Si T, Li N, Lu H, et al. Impact of paliperidone palmitate one-month formulation on relapse prevention in patients with schizophrenia: a post-hoc analysis of a one-year, open-label study stratified by medication adherence. J Psychopharmacol. 2018;32(6):691-701.

42. Ascher-Svanum H, Faries DE, Zhu B, Ernst FR, Swartz MS, Swanson JW. Medication adherence and long-term functional outcomes in the treatment of schizophrenia in usual care. J Clin Psychiatry. 2006;67(3):453-60.

43. Ngui AN, Vasiliadis HM, Tempier R. Factors associated with adherence over time to antipsychotic drug treatment. Clin Epidemiol Glob Health. 2015;3(1):3-9. Available at: https://cegh.net/article/S2213-3984(13)00088-2/ fulltext. Accessed December 27, 2019.

44. Valenstein M, Ganoczy D, McCarthy JF, Myra Kim H, Lee TA, Blow FC. Antipsychotic adherence over time among patients receiving treatment for schizophrenia: a retrospective review. J Clin Psychiatry. 2006;67(10):1542-50. 45. IBM Watson Health, Truven Health Analytics. IBM Micromedix RED BOOK Online. Paliperidone palmitate. Retrieved January 21, 2018. Available at: https://www.ibm.com/products/micromedex-red-book/details. Accessed December 27, 2019.

46. Invega Sustenna (paliperidone palmitate) extended-release injectable suspension, for intramuscular use. Janssen Pharmaceuticals. Revised December 2017. Available at: https://www.accessdata.fda.gov/drugsatfda docs/label/2017/022264s015lbl.pdf. Accessed December 27, 2019

47. Centers for Medicare \& Medicaid Services. Medicaid Drug Rebate Program. Retrieved August 27, 2018. Available at: https://www.medicaid. gov/medicaid/prescription-drugs/medicaid-drug-rebate-program/index. html. Accessed December 27, 2019.

48. Saphris (asenapine) sublingual tablets. Allergan. Revised February 2017. Available at: https://www.accessdata.fda.gov/drugsatfda_docs/ label/2017/022117s022lbl.pdf. Accessed December 27, 2019.

49. Fanapt (iloperidone) tablets, for oral use. Vanda Pharmaceuticals. Revised February 2017. Available at: https://www.accessdata.fda.gov/drugsatfda_docs/ label/2017/022192s018s021lbl.pdf. Accessed December 27, 2019. 
50. Latuda (lurasidone hydrochloride) tablets, for oral use. Sunovion Pharmaceuticals. Revised February 2017. Available at: https://www.accessdata.fda.gov/drugsatfda_docs/label/2017/200603s028lbl.pdf. Accessed December 27, 2019.

51. Invega (paliperidone) extended-release tablets. Janssen Pharmaceuticals Revised August 2017. Available at: https://www.accessdata.fda.gov/drugsatfda_docs/label/2017/021999s034lbl.pdf. Accessed December 27, 2019.

52. Pilon D, Joshi K, Tandon N, et al. Treatment patterns in Medicaid patients with schizophrenia initiated on a first- or second-generation long-acting injectable versus oral antipsychotic. Patient Prefer Adherence. 2017;11:619-29.

53. Healthcare Cost and Utilization Project (HCUP). Weighted national estimates from HCUP National (Nationwide) Inpatient Sample (NIS). 2014. Retrieved January 23, 2018. Available at: https://www.hcup-us.ahrq.gov/ nisoverview.jsp. Accessed December 27, 2019.
54. Heslin KC, Weiss AJ. Hospital readmissions involving psychiatric disorders, 2012. HCUP Statistical Brief \#189. May 2015. Agency for Healthcare Research and Quality, Rockville, MD. Available at: https://www.hcup-us. ahrq.gov/reports/statbriefs/sbl89-Hospital-Readmissions-Psychiatric-Disorders-2012.jsp. Accessed December 27, 2019.

55. Pilon D, Muser E, Emond B, et al. Treatment patterns in Medicaid beneficiaries with schizophrenia reaching stabilized maintenance with oncemonthly paliperidone palmitate therapy. Value Health. 2016;19(3):A196 [abstract]. Available at: https://www.valueinhealthjournal.com/article/S10983015(16)30153-X/fulltext. Accessed December 27, 2019.

56. Dilla T, Ciudad A, Álvarez M. Systematic review of the economic aspects of nonadherence to antipsychotic medication in patients with schizophrenia. Patient Prefer Adherence. 2013;7:275-84.

\section{APPENDIX Probabilistic Sensitivity Analysis: Net Schizophrenia-Related Cost per Nonadherent Patient with a} Recent Relapse Switched to PP1M at Years 2 and 3

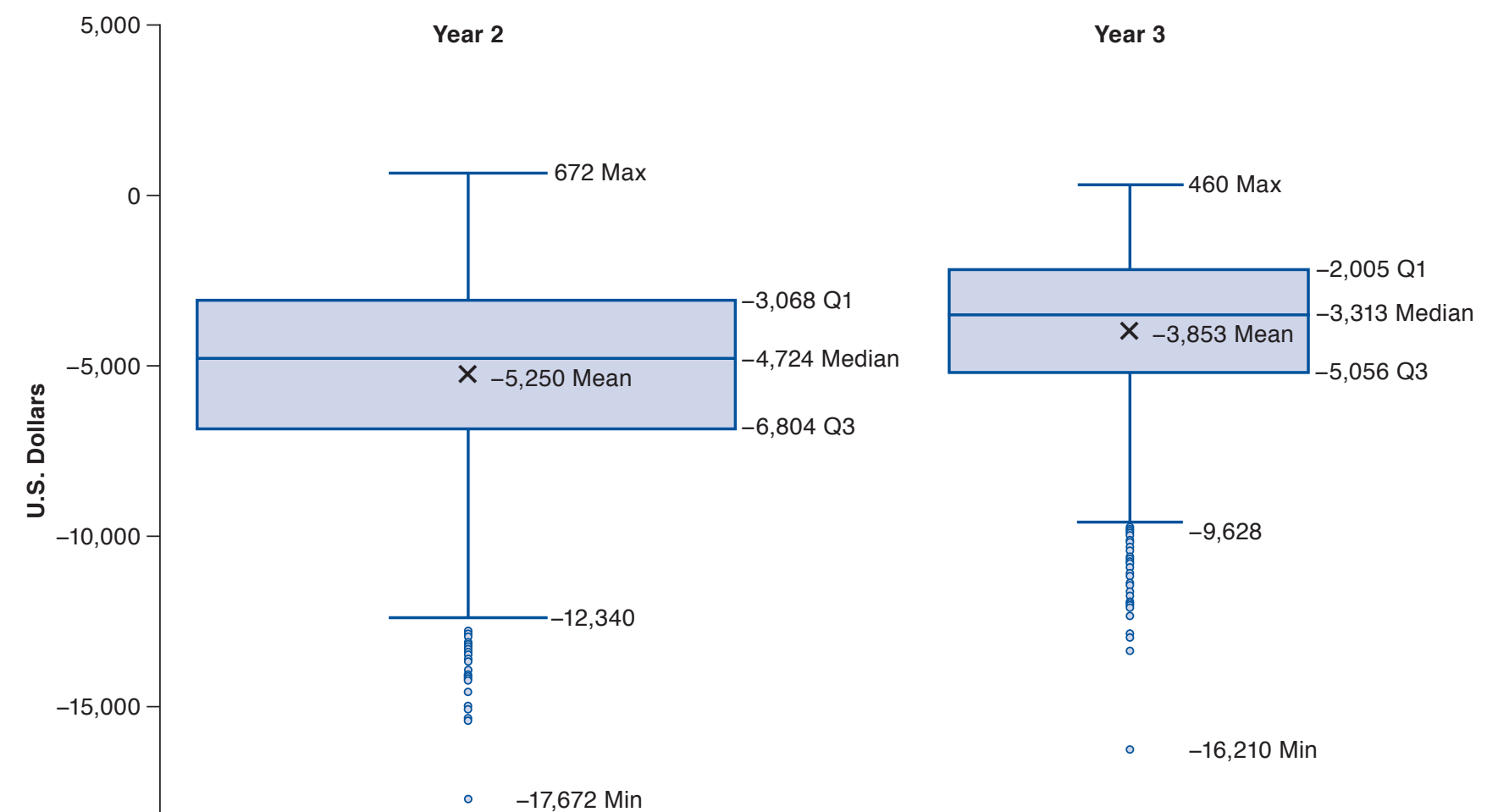

